$\begin{array}{ll} & \text { Etnográfica } \\ \text { etnográfica } & \text { Revista do Centro em Rede de Investigação em }\end{array}$

Antropologia

vol. $16(2) \mid 2012$

Vol. $16(2)$

\title{
Devenir père homosexuel en France : la construction sociale du désir d'enfant
}

Tornar-se pai homossexual em França : a construção social do desejo de ter filhos

\section{Flávio Luiz Tarnovski}

\section{(2) OpenEdition}

Journals

Édition électronique

URL : https://journals.openedition.org/etnografica/1487

DOI : $10.4000 /$ etnografica. 1487

ISSN : 2182-2891

\section{Éditeur}

Centro em Rede de Investigação em Antropologia

\section{Édition imprimée}

Date de publication : 1 juin 2012

Pagination : 247-267

ISSN : 0873-6561

\section{Référence électronique}

Flávio Luiz Tarnovski, « Devenir père homosexuel en France : la construction sociale du désir d'enfant », Etnográfica [En ligne], vol. 16 (2) | 2012, mis en ligne le 26 juin 2012, consulté le 10 février 2022. URL : http://journals.openedition.org/etnografica/1487 ; DOI : https://doi.org/10.4000/ etnografica. 1487

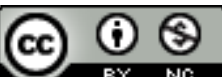

Etnográfica is licensed under a Creative Commons Attribution-NonCommercial 4.0 International License. 


\section{Devenir père homosexuel en France: la construction sociale du désir d'enfant}

\section{Flávio Luiz Tarnovski}

À partir d'une enquête avec des pères homosexuels français membres de l'Association des parents et futurs parents gays et lesbiens (APGL), cet article analyse les contextes sociaux d'émergence du désir d'enfant et certains enjeux liés à l'élaboration du projet parental. L'univers associatif est ici considéré non seulement comme un lieu de visibilité, mais aussi comme un espace de création de nouvelles formes familiales. Ainsi, le désir d'enfant est analysé comme un fait social dont l'expression et la genèse peuvent être situées dans des contextes socioculturels particuliers.

MOTS-CLÉS : désir d'enfant, projet parental, homosexualité, paternité, homoparentalité, famille.

Tornar-se pai homossexual em França: a construção social do desejo de ter filhos - A partir de uma pesquisa com pais homossexuais franceses membros da Associação de Pais e Futuros Pais Gays e Lésbicas (APGL), este artigo analisa os contextos sociais de surgimento do desejo de ter filhos e algumas questões relacionadas com a elaboração do projeto parental. O universo associativo é aqui considerado não apenas como um lugar de visibilidade, mas também como um espaço de criação de novas formas familiares. Neste sentido, o desejo de ter filhos é analisado como um facto social do qual não apenas a expressão mas também a origem podem ser situadas em contextos socioculturais particulares.

PALAVRAS-CHAVE: desejo de ter filhos, projeto parental, homossexualidade, paternidade, homoparentalidade, família.

TARNOVSKI, Flávio Luiz (flaviolt@gmail.com) - Universidade Federal de Mato Grosso, Cuiabá, Brasil 
LES FAMILLES CONSTITUÉES PAR LES GAYS ET LES LESBIENNES FONT désormais partie du paysage familial contemporain de la plupart des pays occidentaux. Leur reconnaissance juridique fait encore l'objet de controverses et de disputes politiques, mais leur existence est déjà un fait social décrit et analysé par plusieurs enquêtes (Lewin 1993, 2009; Fournier 2003; Gross 2005; Cadoret 2002; Cadoret et al. 2006; Gratton 2006; Grossi et al. 2007; Descoutures 2008; Herbrand 2009). L'importance sociale de ces configurations familiales n'étant plus en question, il s'agit avant tout d'élargir notre compréhension de cette nouvelle réalité ethnographique constituée par les multiples parentalités homosexuelles (Schneider 1997; Favret-Saada 1999; Almeida 2010). Dans le contexte social précis de la société française, cet article étudie la construction du désir d'enfant et du projet parental chez des hommes qui s'assument comme homosexuels et sont membres de l'Association des parents et futurs parents gays et lesbiens (APGL).

A partir d'observations participantes et d'entretiens réalisés avec 23 pères homosexuels à Paris et à Toulouse dans le cadre d'une thèse de doctorat en anthropologie sociale (Tarnovski 2010), j'analyse les contextes sociaux d'émergence du désir d'enfant et les modalités de sa réalisation à travers un projet parental. ${ }^{1}$ En France, la visibilité des familles homoparentales ${ }^{2}$ est indissociable de l'action de l'APGL, fondée en 1986, forte, aujourd'hui, de plus de 1600 adhérents répartis entre différentes configurations familiales créées à partir de la recomposition homoparentale, de l'adoption, de la coparentalité, de l'insémination avec donneur ou de la gestation pour autrui.

\section{LE DÉSIR D’ENFANT COMME NORME SOCIALE}

Avoir un enfant aujourd'hui n'a pas le même sens qu'il y a trente ou cinquante ans. Progressivement, une nouvelle norme s'est imposée, celle du désir d'enfant. Selon Delaisi de Parseval (2008: 43): “L'expression même de 'désir d'enfant' est très récente, précisément liée à l'ère contraceptive". Selon cette auteure, avant l'apparition et la diffusion des méthodes contraceptives ce désir n'avait pas besoin d'être exprimé, car c'était une évidence que d'avoir des enfants et, donc, pour les générations précédentes, il n'existait aucune nécessité de le déclarer publiquement. De nos jours, la fécondité est pensée comme un projet personnel qui exige constamment des choix de la part du sujet (Bozon 2002; Tain 2005). Avoir un enfant n'est plus une étape de la trajectoire du couple qui va de soi :il faut d'abord y réfléchir, penser au meilleur moment pour en avoir, combien en avoir, avec qui... Cela ne veut pas dire qu'avoir une descendance

l Cette recherche a été financée par une bourse de la Capes/Ministère de l'Éducation brésilien.

2 La notion d'homoparentalité a été créée par l'APGL pour désigner les familles dont l'un des parents au moins s'assume comme homosexuel (Gross 2005). 
soit devenu un enjeu moins central pour les couples. En réalité, moins que de devoir assurer un descendant à la lignée, c'est l'enfant en lui-même qui compte le plus aujourd'hui, ce qui impose de réfléchir à son arrivée, à la place qu'il va occuper, aux conditions matérielles nécessaires pour bien l'accueillir. Par ailleurs, le nombre croissant d'unions libres a fait que, désormais, la naissance d'un enfant marque l'entrée en famille du couple, passage auparavant assuré par l'institution du mariage (Théry 1998).

On peut choisir de ne pas avoir d'enfants soit parce que les bonnes conditions ne sont pas réunies, soit parce que le bon partenaire n’a pas été trouvé. Mais ce choix doit encore être justifié socialement. Dans ce contexte, et différemment de ce qui est habituellement le cas des couples hétérosexuels, pour les homosexuels, c'est l'existence du désir qui est considérée comme problématique et non pas son absence. On n'attend pas d'eux (même si cela peut devenir de moins en moins vrai actuellement) qu'ils aient des enfants (surtout par les voies dites "naturelles”). L'étrangeté que suscite le désir d'enfant manifesté par des gays et des lesbiennes, surtout lorsqu'il s'agit du premier, révèle que tous ne sont pas égaux face à ce désir, même si, comme le souligne Godelier (2004), l'évolution historique de la valeur de l'enfant peut expliquer son expression chez les homosexuels.

Pour interroger le désir d'enfant des gays et des lesbiennes, il ne faut en aucun cas souscrire à une vision selon laquelle l'homosexualité y serait d'emblée contradictoire ou incompatible, car tout désir d'enfant doit être examiné à partir d'un point de vue anthropologique. Par ailleurs, il ne s'agit pas non plus de naturaliser ce désir en affirmant que les homosexuels qui ne l'expriment pas "renonceraient" ou auraient pendant longtemps "renoncé" à l'idée d'avoir d'enfants. ${ }^{3}$ La compréhension des conditions sociales de manifestation et d'expression du désir d'enfant chez les homosexuels exige une analyse qui ne s'enferme pas dans les termes de cette dichotomie selon laquelle ce désir serait soit "artificiel", soit "naturel" chez eux.

Les questions que soulève le désir d'enfant manifesté par des hommes s'assumant comme homosexuels sont les mêmes que pourrait susciter le désir d'enfant exprimé par n'importe quelle personne. Quelle est sa signification? Pourquoi a-t-on des enfants et à quel moment de sa trajectoire de vie? Quelles conditions doivent être réunies pour que le désir d'enfant puisse s'exprimer et se réaliser? Faut-il toujours que la naissance d'un enfant soit précédée d'un désir?

3 Ce raisonnement est également utilisé pour expliquer les trajectoires d'hommes qui se sont assumés comme homosexuels après une période de conjugalité hétérosexuelle. D’après ce discours, leur paternité serait le résultat d'un “renoncement” à l'homosexualité, motivé par un fort désir d'enfant. C'est la même logique qui est à l'œuvre, mais avec une inversion des termes. Pour une critique de ce discours, voir Tarnovski (2012). 
L'une des spécificités du désir d'enfant homosexuel, par rapport à son homologue hétérosexuel, réside dans le degré de réflexivité que suscite le premier. Sandrine Fournier, dans son analyse sur les familles homoparentales de San Francisco, affirme: "Le choix de créer une famille, contre toute attente, se vit [...] comme l'expression 'par excellence' d'un choix personnel" (2003: 63). Ce choix n'est néanmoins possible qu'à partir du moment où il s'offre comme une éventualité plausible, c'est-à-dire lorsque l'idée de fonder une famille peut être envisagée comme une alternative réalisable par des homosexuels, qu'ils soient en couple ou célibataires. Dans le contexte actuel, vouloir devenir père lorsqu'on s'assume comme homosexuel exige un travail réflexif, parfois intense, sur le désir d'enfant. La clarté du contenu qu'on lui attribue est d'autant plus grande et nécessaire qu'un tel désir n'est pas conforté par des modèles socialement cristallisés.

Dans sa thèse sur la paternité gay en France, Emmanuel Gratton (2006) propose une analyse approfondie du désir d'enfant chez des hommes homosexuels déclarés dans ses dimensions psychologiques et sociologiques. Selon lui, si le désir d'enfant n'était pas, jusqu'à une date récente, exprimé par des homosexuels, c'est que la société y faisait obstacle, et les homosexuels eux-mêmes, ne trouvant que des barrières à la réalisation de ce désir, se résignaient à une vie sans enfants. Gratton (2006: 23) ajoute: "S'affirmer homosexuel procède souvent d'une forme de renoncement anticipé à la parentalité". D'après lui, les homosexuels qui veulent être parents devraient "s'affranchir" d'un ensemble de normes qui les "assujettissent" en tant qu'homosexuels et qui font barrage à la manifestation d'un désir d'enfant.

L'analyse de Gratton montre que le désir d'enfant exprimé par des homosexuels peut avoir des motivations très variées, mais, finalement, il ne met sa dimension sociale en valeur que dans ses aspects négatifs, comme dans l'idée selon laquelle les homosexuels devraient "s'affranchir" des normes sociales pour devenir parents, en surmontant les "obstacles" imposés par la société à la réalisation de leur désir d'enfant. Le modèle analytique construit par Gratton permet effectivement de comprendre les difficultés affrontées par les homosexuels voulant devenir pères. Cependant, l'universalité du désir d'enfant y apparaît comme une donnée précédant les analyses. Son existence n'est pas interrogée.

Dans les analyses qui suivront, je propose une autre approche pour comprendre le désir d'enfant exprimé par mes interlocuteurs. Au lieu de le considérer comme un fait universel, dont seules les modalités de réalisation seraient socialement déterminées, je suggère que ce désir est inséparable des contextes socioculturels qui l'investissent d'un sens subjectif et politique. 


\section{“CELA A ÉTÉ UNE RÉVÉLATION": VOULOIR (ET POUVOIR) ÊTRE PÈRE EN TANT QU'HOMOSEXUEL}

Christophe, 66 ans, enseignant retraité, a trois enfants, une fille de 32 ans et deux fils de 11 et 12 ans. ${ }^{4}$ Sa trajectoire a été marquée par les transformations récentes dans le domaine de la sexualité et de la famille. Tout en se sachant homosexuel, Christophe s'est marié à l'âge de 32 ans dans le seul but d'avoir un enfant. A l'époque, lui et son épouse faisaient des études de troisième cycle et c'est dans ce cadre qu'ils se sont rencontrés. Ils ont vécu ensemble pendant deux ans, jusqu'à ce qu'elle tombe enceinte et demande le divorce. Après la naissance de l'enfant, elle lui a demandé de renoncer à ses droits en tant que père, mais il a refusé tout accord dans ce sens. Même si leurs rapports sont tendus, Christophe arrive à voir sa fille régulièrement. Quelques années plus tard, il a entretenu une relation avec une autre femme qui est aussi tombée enceinte mais, sans le consulter, a décidé de ne pas garder l'enfant. Christophe est passé par une phase de dépression profonde et a renoué avec un ancien amant, Charles, avec qui il est en couple depuis plus de vingt ans. En raison des difficultés rencontrées en tant que père homosexuel divorcé, Christophe a adhéré à l'APGL peu de temps après sa fondation à Paris. Des réunions conviviales étaient organisées chez lui et c'est là qu'il a connu les femmes avec lesquelles il allait avoir deux fils à travers des projets de coparentalité. ${ }^{5}$ La mère de l'aîné vit dans le Sud de la France et il voit son fils régulièrement. Avec la mère de son deuxième fils les choses ne se passent pas aussi bien et il a dû entamer une procédure judiciaire pour pouvoir voir son enfant. Il a eu gain de cause, mais ne peut le rencontrer qu'en visite surveillée.

André, 31 ans, enseignant, considère qu'il a toujours voulu fonder une famille et avoir des enfants, dès son jeune âge. Au fil des années, cette perspective serait progressivement devenue une certitude, jusqu'à ce qu'il "découvre" qu'il "était homosexuel". La prise de conscience de l'attirance homosexuelle l'aurait perturbé par rapport à la volonté d'avoir des enfants, mais "ne l'a jamais remise en cause”. A l'époque, c'est-à-dire pendant son adolescence, l'idée de faire un enfant avec une femme lesbienne ne se posait pas comme une possibilité. La seule solution qu'il pouvait imaginer, c'était de se marier avec une femme tout en se sachant homosexuel. Après un séjour à l'étranger pour terminer ses études, André a trouvé un poste dans une ville de province où il s'est installé. Vers l'âge de 26 ans, un des ses amis lui parle de la coparentalité entre gays et lesbiennes et lui apprend l'existence de l'APGL. Sans plus

4 Les prénoms ont été changés.

5 La coparentalité consiste à s'associer avec un partenaire de l'autre sexe, selon des modalités très variées, pour faire un enfant, avec ou sans rapports sexuels, et l'élever en alternance. La conjugalité et la filiation se trouvent ainsi dissociées et l'enfant peut appartenir, dès sa naissance et de façon simultanée, à deux unités domestiques et familiales différentes (Cadoret 2002; Tarnovski 2011 ). 
attendre, André y adhère pour connaître la réalité des familles ainsi constituées. Un garçon est né, quatre ans après, fruit d'une coparentalité avec un couple de femmes.

Par rapport à celui d'autres hommes plus âgés, l'exemple d'André est révélateur des changements récents concernant l'accès à la paternité par des homosexuels. La fille aînée de Christophe, par exemple, a été conçue à une époque où l'homosexualité ne pouvait être vécue au grand jour, comme cela peut être le cas aujourd'hui, ou en tout cas moins facilement, et où la parentalité ne se dissociait pas de la conjugalité, contrairement à ce qui se passe dans la plupart des coparentalités entre gays et lesbiennes ayant commencé à se constituer à une époque plus récente. Le parcours de Christophe témoigne d'une époque où, pour pouvoir devenir père, même en s'assumant plus ou moins ouvertement comme homosexuel, il était moins facile qu'aujourd'hui de passer outre une période de fréquentation hétérosexuelle avec la mère, c'est-à-dire de dissocier la parentalité de la conjugalité. Même quand l'idée d'un accord était implicite, comme cela semble avoir été le cas pour le mariage de Christophe, ses termes n'étaient pas discutés de façon explicite, donnant lieu à des situations difficilement gérables par la suite.

Ce qui distingue l'époque actuelle de celle d'il y a trente ou quarante ans n'est pas tant une "évolution" des pratiques ayant conduit à la disparition des plus anciennes et "dépassées", qu'un élargissement du champ des choix possibles qui s'offrent aux hommes voulant devenir pères tout en s'assumant homosexuels. Il y a d'un côté la visibilité croissante de la notion même d' "homoparentalité", notamment à travers l'action d'associations de parents homosexuels, et de l'autre, un changement plus général dans le domaine du mariage et de la famille. Les unions libres n'ont cessé d'augmenter ces dernières décennies, et les lois concernant l'établissement de la filiation hors mariage, la reconnaissance paternelle et le partage de l'autorité parentale se sont adaptées à cette nouvelle réalité. Dans le contexte français, l'APGL a joué et joue encore un rôle important non seulement en rendant visibles les parentalités homosexuelles, mais aussi, et de façon plus importante, en devenant un lieu de "production" de ces mêmes formes familiales. En tant qu'espace de rassemblement, rencontre, échange, réflexion, et grâce à la synergie des individus qui en font partie et au concours de différentes compétences et expériences, cette association s'est constituée en un lieu de création d'arrangements familiaux, d'élaboration et de mise en pratique de projets parentaux. Christophe, par exemple, fait partie de ceux qui, en expérimentant de nouvelles formes familiales comme la coparentalité, ont élargi le champ des possibilités de la désormais nommée "homoparentalité". On pourrait affirmer qu'en France, comme dans d'autres pays du monde occidental, ne pas avoir d'enfants parce qu'on est homosexuel, est de moins en moins considéré comme un "destin" et devient progressivement, bien que 
parfois abruptement, un "choix". ${ }^{6}$ Cela ressort clairement du récit de certains pères lorsqu'ils décrivent les circonstances qui les ont sensibilisés à un désir d'enfant possible.

Pour Jean, 43 ans, ingénieur, les origines de son désir d'enfant remontent à une date très précise, lorsqu'il avait 35 ans, plus exactement un samedi matin, jour de la gay pride, dans une conversation avec son compagnon d'alors, Julien. Le thème du défilé était justement l'homoparentalité, et c'était la première fois qu'il en prenait connaissance. Il se rappelle avoir trouvé le sujet "marrant", d'autant plus qu'il ne se sentait pas concerné. En parlant avec Julien, celui-ci lui aurait avoué qu'il avait très envie d'avoir un enfant. Selon Jean: "C'était une fatalité, comme une évidence que je n'avais pas remise en cause et donc cette discussion avec mon copain me l'a fait au fond remettre en cause. [...] Et donc, d'un seul coup, je me suis senti concerné". Le fait que son compagnon ait la volonté d'avoir un enfant l'interpelle et favorise un travail réflexif sur son propre désir. A partir du moment où la paternité devient pour lui une réalité possible, le fait de ne pas être père ne constitue plus une "évidence" mais un choix. Désormais, c'est l'absence de désir d'enfant qui devient problématique, dans la mesure où elle commence à être perçue comme le résultat d'une forme d' "aveuglement" et non pas comme une réalité en soi. Peu de temps après, Jean irait à une rencontre de l'APGL, qu'il ne connaissait pas jusqu'alors, pour connaître une femme qui accepterait de faire un enfant avec lui. Cinq ans après naîtrait une petite fille, dont Julien serait le parrain. ${ }^{7}$

Vincent, 42 ans, infirmier, a pris connaissance de l'existence des familles homoparentales au moment de la réalisation d'un mémoire, préparé dans le cadre d'une formation universitaire. Ce sujet a été proposé par une collègue, homosexuelle elle aussi, avec qui il a rédigé le mémoire. Jusqu'alors, et cela s'est passé vers 1999, Vincent n'avait jamais entendu parler des familles homoparentales. La rencontre avec l'APGL a été pour lui une "révélation": "Je suis revenu à la maison et j'ai dit 'Victor, tu ne vas pas imaginer ce que je viens de voir'". Pour son compagnon, âgé de 40 ans, infirmier, l'idée que des homosexuels aient des enfants paraissait complètement "incroyable", "farfelue". C'était la première fois que cette question était abordée dans leur couple. Vincent ne se l'était jamais posée, puisque pour lui ce n'était même pas possible:

6 L'un des pères interviewés m'a raconté que certaines relations d'amitié ont été bouleversées après l'arrivée de l'enfant dans la mesure où la réalité de l'homoparentalité a confronté ses amis homosexuels à des choix qu'ils auraient pu faire dans leur trajectoire de vie.

7 À ce moment-là, Julien et Jean n'étaient plus en couple. Julien, 40 ans, graphiste, attendrait encore quelques années avant de se sentir "prêt” à mettre en pratique son propre projet d'enfant. Le fait que Jean ait réussi à le réaliser n’a pas été sans importance pour que Julien puisse se sentir motivé à devenir père à son tour. 
“Je pense que le fait d'avoir vécu ma vie d'homo dès l'âge de 18 ans et d'avoir parlé à mes parents assez tôt, de fait, ça a étouffé tout désir d'enfant possible. Et pour être honnête, à l'époque, je l'aurais vu comme une contrainte. On voyageait, on faisait des trucs, un enfant là nous aurait posé problème même. C'était même pas évocable, on ne s'est jamais posé la question. Par contre, du moment où on a vu que c'était possible, ça a été vraiment une révélation, pour moi, en tout cas".

A la suite de cette “révélation”, il a adhéré à l'APGL avec son compagnon, mais ce dernier est resté plus en retrait par rapport à la possibilité de réalisation d'un projet parental. Vincent, de son côté, était décidé à le faire. Moins de deux ans après, il avait adopté un garçon et en a eu un deuxième dans le cadre d'une coparentalité avec un couple de femmes. Confronté à la réalité de la parentalité, Victor a finalement voulu participer à l'agrandissement de la famille. Actuellement, ce couple a quatre enfants, trois garçons et une fille.

Dans le récit de Vincent, comme dans d'autres, ne pas avoir d'enfants n'était pas une source de souffrance. ${ }^{8}$ La réponse récurrente, “je ne me suis jamais posé la question", indique qu'être père en tant qu'homosexuel n'était pas considéré comme une alternative possible, dans la mesure où cette conjugaison d'expériences ne relevait pas de l'évidence. Comme le signale Vincent dans l'extrait supra, le moment de la trajectoire de vie à partir duquel l'individu s'assume comme homosexuel peut avoir une incidence sur son éventuel désir d'être père. Son récit suggère que, jusqu'à une époque récente, plus on s'assumerait homosexuel tôt, plus on aurait tendance à ne pas se sentir concerné par l'idée d'avoir des enfants.

Si, pour certains, le désir d'enfant a été motivé suite à un contact avec la notion d'homoparentalité, pour d'autres la question s'est posée presque inversement. En effet, quelques-uns ont été confrontés à la possibilité concrète d'avoir un enfant en raison de l'invitation d'une amie désireuse de devenir mère. Georges et Guillaume, par exemple, se sont trouvés face à un choix à faire, qui n'était pas forcément à l'ordre du jour. Georges, 42 ans, cadre dans le secteur privé, n'avait fait aucune démarche pour devenir père jusqu'à ce qu'une amie, hétérosexuelle, lui propose une coparentalité “à trois” avec son compagnon, Guillaume. Le sujet le “travaillait”, puisqu'il était alors déjà adhérent à l’APGL. Guillaume, 45 ans, élu municipal, de son côté, ne s’était jamais posé

8 A ce sujet, il faut considérer le biais que comporte mon échantillon, dans la mesure où je n'ai pas interviewé des hommes n'ayant pas pu réaliser un désir de paternité. En même temps, cette souffrance peut être de deux ordres, selon le moment où elle est ressentie, avant ou après une tentative non réussie d'avoir un enfant, souffrance qui peut alors soit être associée à l'idée de "renoncement" de la paternité qu'impliquerait la reconnaissance de soi comme homosexuel, soit être liée à des difficultés concrètes de réalisation d'un projet parental (pour cause d'infertilité ou face à des refus successifs de l'agrément pour l'adoption, par exemple). 
la moindre question sur l'éventualité de devenir père: "Ce n'était pas dans le champ de l'imaginable et puis il n'y avait aucune référence". Leur amie étend sa proposition aux deux, l'idée étant d'avoir un enfant avec chacun. Georges étant plus sensible à la question du désir d'enfant, c'est lui qui devient père en premier.

Ce qui est particulier chez les pères interrogés c'est leur capacité à pouvoir situer précisément soit le jour où ce désir est apparu comme possible, pour ceux qui ne l'avaient jamais envisagé auparavant, soit le moment à partir duquel sa réalisation, en tant qu'homosexuels, ne paraissait plus si étrange que jusqu'alors. Des événements ou des situations passées sont découpées et réorganisées à l'intérieur d'une trame narrative, donnant un sens nouveau à leur intention, et/ou à la possibilité de sa mise en acte, d'avoir un enfant et d'être pères. Dans le récit de Jean, le jour où l'idée d'homoparentalité lui est apparue comme une possibilité concrète est décrit comme "un samedi matin, jour de la gay pride". Julien, son compagnon de l'époque, mentionne "un dimanche matin, ensoleillé, assis devant la fenêtre". La capacité à pouvoir situer le point précis d'origine du désir d'enfant révèle que sa manifestation n'est pas considérée comme allant de soi, ce qui est à mettre en relation avec des représentations de genre marquant ce désir du signe de la féminité (Fournier 2003).

Virginie Descoutures (2008), dans sa thèse de doctorat sur les mères lesbiennes en France, met l'accent sur les difficultés de certaines femmes à envisager la maternité après s'être assumées comme lesbiennes. Dans son analyse, Descoutures considère que la maternité chez les lesbiennes est une forme de "transgression", par rapport à une "norme sociale" selon laquelle "les homosexuel(le)s n'ont pas d'enfants, ne peuvent pas en avoir" (Descoutures 2008: 140). Il n'en reste pas moins que, chez les lesbiennes, le non-désir d'enfant semble moins imprégné d'un sentiment d'évidence que chez les gays, ce qui s'explique par le rapport différencié qu'auraient les hommes et les femmes face à la procréation et à la parentalité. En référence à un autre ensemble de normes, celles concernant le genre, le désir d'être mère paraît moins dépendant de l'orientation sexuelle et du statut conjugal que le désir d'être père. Ce n'est que récemment qu'un désir d'enfant masculin commence à s'exprimer de façon autonome, sans s'associer à un désir de maternité, et plus visiblement chez les hommes qui s'assument comme homosexuels.

Mais, même si le désir d'enfant est, pour ainsi dire, animé par une prise de conscience parfois soudaine de l'existence de familles homoparentales, il n'en est pas moins central pour l'individu et la façon dont il se projette dans l'avenir. En même temps, le fait que la visibilité des familles homoparentales ait mis la paternité dans l'horizon des possibilités offertes à l'individu qui s'assume comme homosexuel n'est pas une raison suffisante pour que tous ceux dans cette situation veuillent avoir des enfants. La conjugaison positive de l'homosexualité avec la paternité peut, certes, rendre la volonté de fonder une famille 
moins conflictuelle, tant subjectivement que socialement. Néanmoins, pour que la personne se sente concernée par la question, d'autres facteurs peuvent entrer en jeu, comme la relation de couple.

\section{“ET SI ON AVAIT UN ENFANT ? ”:DÉSIR D’ENFANT ET CONJUGALITÉ}

Malgré le fait que les hommes membres de l'APGL soient plus souvent en couple que la moyenne de la population gay en France, ils sont moins nombreux que les femmes à affirmer que le projet d'avoir un enfant émane du couple. Selon Martine Gross (2006), les compagnes des mères se situeraient plus souvent que les compagnons des pères comme "second parent". Dans mon enquête, certains pères ont affirmé que le désir d'enfant a pris forme au sein du couple.

Cela a été le cas de Bernard, 37 ans, orthophoniste. Avant de rencontrer son actuel compagnon, il avait déjà eu une relation de couple qui avait duré dix ans. Néanmoins, il ne se voyait pas avoir des enfants avec son ancien partenaire. Avec Bruno, 45 ans, médecin, il se sent plus rassuré et envisage alors d'en avoir un "avec" lui. Dans son cas, la stabilité de la vie de couple et les qualités qu'il perçoit chez son compagnon sont à l'origine de son désir d'enfant. Malgré la surprise initiale de Bruno devant la proposition de Bernard, c'est finalement lui qui sera le "père biologique" d'une petite fille conçue dans le cadre d'une coparentalité. Bruno était le plus âgé, ce qui a joué un rôle dans le choix de qui serait le géniteur. Parallèlement, la rencontre avec le couple de femmes avec lequel ils ont mis en œuvre la coparentalité a également influencé ce choix, en raison d'affinités qui se seraient créées entre la future mère et Bruno. En tout cas, l'arrivée de l'enfant s'inscrit pour eux dans une dynamique de couple, marquée par un investissement conjoint dans le projet de coparentalité et dans l'exercice de la parentalité. Lorsque nous nous sommes rencontrés, Bernard était engagé dans une deuxième coparentalité, avec un autre couple de femmes, mais les premiers essais de fécondation s'étaient montrés infructueux. Dans l'idée que se font Bernard et Bruno de l'égalité à l'intérieur de leur couple, la paternité de Bernard permettrait de compenser une certaine asymétrie créée par la paternité de Bruno, dans la mesure où l'un "donnerait" un enfant à l'autre. Selon Bernard, “[...] c'est plutôt Bruno qui aimerait avoir [de moi] un enfant".

Dans le cas de Sébastien, 50 ans, médecin, le désir d'enfant est également apparu dans un cadre conjugal, mais non sans tensions. Après quelques années de vie de couple, son compagnon a trouvé qu'il "manquait quelque chose". D'après celui-ci, ils avaient tous les deux une profession, un appartement, de la stabilité, mais ils n'étaient pas une famille. A écouter Sébastien, pour son compagnon, "un couple sans enfant n'est pas une famille". Celui-ci aurait ajouté que leur vie "n'était pas totalement réalisée sans enfant". Au début, Sébastien considérait inutile toute discussion sur le sujet, car avoir un enfant en tant 
que couple homosexuel lui paraissait un "rêve impossible". Toutefois, et face à l'insistance de son compagnon, il accepte d'aller à une réunion de l'APGL. Une fois sur place, il rencontre un des adhérents de l'association qui lui dit qu'il allait bientôt être père. Cette déclaration l'a fortement affecté, d'autant plus qu'un mois plus tard il croise cette même personne lors de la gay pride qui lui dit: "Ça y est ! Je suis papa”. En l'espace de trois mois, d'avril à juin, un changement radical s'est produit dans la façon dont Sébastien pouvait considérer la parentalité chez un couple homosexuel: "Ce qui me semblait une invention de l'esprit devenait une réalité que l'on pouvait toucher, en trois, quatre mois. Ça a été très, très fort, ça a été un déclic important parce que ça a montré que c'était possible".

Après s'être mis d'accord sur l'idée d'avoir des enfants, Sébastien et son compagnon ont entrepris des démarches, mais dans un premier temps ils ont, chacun de son côté, mené des projets individuels de paternité. Sébastien privilégiait l'idée d'une coparentalité, tandis que son compagnon s'était tourné vers une gestation pour autrui traditionnelle, aux États-Unis. ${ }^{9}$ Mais, comme ni l'un ni l'autre n'arrivait à avancer dans la réalisation de leur projet de paternité, ils ont décidé de concentrer leurs efforts sur la GPA. Ainsi, même si le désir d'enfant a pris forme à l'intérieur du couple, sa réalisation a été initialement envisagée à travers la mise en place des projets individualisés de paternité. L'enfant arrivé, Sébastien se considère "père à cent pour cent", mais cette implication conjointe dans la parentalité semble avoir été plutôt le résultat d'une construction progressive à l'intérieur du couple qu'une donnée de départ. A cet égard, le contraste avec le couple formé par Thierry et Thomas permettra peut-être d'éclairer les différentes implications que peut avoir la conjugalité dans les multiples agencements de la parentalité.

Ingénieurs, tous deux âgés de 31 ans, Thierry et Thomas se sont connus à l'université, il y a douze ans. Ils n'ont pas attendu longtemps pour vivre ensemble. Après trois mois de fréquentation, encore à l'université, Thierry a loué un appartement voisin de celui de Thomas. Peu à peu les copains d'études ont été mis au courant de leur relation. Deux ans après, ils ont décidé d'habiter un seul appartement, ce qui a coïncidé avec la révélation de leur union aux familles respectives. A cette époque, en 1997, ils pensaient déjà à adopter des enfants, mais l'idée leur paraissait peu crédible. Après un stage réalisé aux Pays-Bas, pendant lequel ils ont pu connaître des familles constituées par des

9 D’après Delaisi de Parseval et Collard, la gestation pour autrui (GPA), appelée aussi “maternité de substitution”, “[...] est une pratique par laquelle une femme porte un foetus, et poursuit la grossesse jusqu'à la naissance de l'enfant avec l'intention de transférer ensuite ses droits et devoirs parentaux $\mathrm{au}(\mathrm{x})$ parent(s) d'intention" (2007: 29). Elle peut avoir deux formes, l'une où la gestatrice n'est pas la mère génétique de l'enfant, et l'autre, traditionnelle, où elle est inséminée avec le sperme du père d'intention. Voir aussi Gross et Mehl (2011). 
homosexuels, ils ont réfléchi à la possibilité d'y retourner pour adopter. Néanmoins, à cette époque leurs préoccupations étaient principalement tournées vers la fin des études et leur insertion professionnelle. Le projet d'avoir des enfants n'est revenu à l'ordre du jour qu'une fois cette stabilité conquise. Pour Thierry, l'idée de fonder une famille avec des enfants est apparue comme une conséquence logique de la vie de couple: "C'est venu un peu dans l'ordre des choses, comme pour n'importe qui”. D'après lui, ce n'est pas un projet qui est apparu "d'un seul coup", mais le résultat d'une construction progressive. Contrairement à ce qui s'est passé avec Sébastien et son compagnon, ici la conjugalité et la parentalité ont été pensées comme indissociables dès le début de leur réflexion sur un possible projet en vue d'avoir des enfants. En témoigne la façon choisie pour mettre le projet en pratique, une GPA. Le choix de celui qui allait être le géniteur des enfants et, par conséquent, le père juridiquement reconnu, a été réalisé selon des critères pratiques, et il ne répondait pas à un désir d'enfant plus prononcé. Tous deux ont convenu que Thierry serait le géniteur, en raison de ses connaissances de la langue anglaise, ce qui faciliterait les contacts avec les génitrices potentielles et les avocats aux États-Unis. Ils pensent, à posteriori, que ce choix a, en fin de comptes, compensé un certain déséquilibre concernant les rapports avec les familles respectives. Thierry a des rapports très limités avec sa famille, qui se résument aux contacts qu'il a avec sa mère et avec deux cousines, l'une d'entre elles étant lesbienne. Il est fils unique de parents divorcés et ne voit pas très souvent son père. D'ailleurs, il croyait que la venue d'enfants lui aurait permis de s'en rapprocher, ce qui n'a pas été le cas. En revanche, du côté de Thomas, la famille a été très présente et a accueilli avec joie la venue de leurs enfants, et ce indépendamment du fait que Thomas n’a aucun lien génétique ou légal avec les enfants. Ainsi, bien que Thierry soit le père légal des enfants, il n'a pas, selon ses propres mots, “à qui offrir ce lien". En revanche, la réaction des parents de Thomas, qui ont commencé à s'impliquer dès le troisième mois de grossesse, en demandant toujours des nouvelles, les a heureusement surpris.

Bien qu'il puisse prendre forme à l'intérieur du couple, dans certains cas, le désir d'enfant n'a été exprimé que par l'un des partenaires. Vincent, par exemple, l'a réalisé en adoptant un garçon et en ayant un autre dans le cadre d'une coparentalité, mais sans que son compagnon soit partie prenante d'aucun de ces projets parentaux. Ce n'est qu'après l'arrivée des enfants que Victor a commencé à s'impliquer en tant que parent et à envisager, lui aussi, d'avoir des enfants. L'écart qui pouvait exister entre l'un et l'autre par rapport au désir d'enfant s'est rétréci avec le temps, la présence des enfants dans le quotidien domestique ayant sans doute joué un rôle important dans ce changement progressif.

Les cas analysés montrent que même dans les situations où la conjugalité est à l'origine de la volonté d'avoir un enfant, les partenaires ne s'investissent 
pas toujours de la même manière dans ce projet, ni ne veulent systématiquement occuper une position parentale symétrique à celle du père légal. ${ }^{10}$ Lorsque la volonté d'avoir des enfants est considérée comme un symbole de l'amour conjugal, différentes formes de symétrie entre les positions parentales des partenaires peuvent être mises en place par les couples, comme pour compenser les inégalités de statuts parentaux. Cette symétrie peut se traduire par une dévalorisation de l'importance de la "paternité génétique", comme chez Thierry et Thomas, ou par un échange de statuts, comme chez Bernard et Bruno. Dans ce dernier cas, l'idée que chacun soit le géniteur et le père légal d'un enfant a été la solution choisie pour produire un équilibre à l'intérieur du couple concernant les statuts parentaux. Néanmoins, dans la coparentalité les enfants doivent être "partagés" avec la mère et sa compagne, ce qui crée une asymétrie entre les couples. Le choix de la GPA ou de l'adoption, au contraire, permet une association plus étroite entre conjugalité et parentalité. Gross et Mehl (201 l) identifient, dans le contexte de la GPA, une tendance de la part des couples d'hommes à l'exercice conjoint de la responsabilité éducative au quotidien, car les enfants ne sont pas partagés avec d'autres "partenaires de l'enfantement".

Nous avons vu que la prise de connaissance de l'existence des familles homoparentales peut, dans un premier temps, déclencher chez l'individu qui s'assume comme homosexuel le désir de devenir parent et que le fait d'être en couple favorise, dans certains cas, l'expression de ce désir, mais sa réalisation exige d'abord que son porteur puisse le transformer en projet.

\section{LE DÉSIR RÉALISÉ OU SA MÉTAMORPHOSE EN PROJET: QUEL TYPE DE FAMILLE CHOISIR?}

Différemment de ce qui se passe habituellement chez un couple hétérosexuel sans problème de fertilité, pour un couple ou un individu homosexuel, le temps entre le moment où l'on pense plus sérieusement à avoir un enfant et le jour de son arrivée peut largement dépasser les neuf mois. Les homosexuels décidés à avoir un enfant doivent obligatoirement réfléchir aux moyens qu'ils choisiront pour devenir parents. Le désir d'enfant, pour pouvoir se réaliser, doit être envisagé dans ses aspects concrets, prenant ainsi la forme d'un projet parental dont le contenu et l'importance dépendront du mode de procréation et de la forme familiale envisagés. Comme tout couple ou toute personne voulant avoir un enfant, ils se posent la question des conditions jugées nécessaires pour être en mesure de l'accueillir. Mais à la différence de la plupart des couples, les homosexuels dans cette situation doivent non seulement réunir les moyens pour 
élever un enfant dans des conditions considérées comme satisfaisantes, mais être capables de mobiliser des ressources relationnelles et matérielles pour, en premier lieu, pouvoir réaliser le projet parental. ${ }^{11}$

Pour les sujets rencontrés, la construction du projet a d'abord impliqué la considération des différentes alternatives possibles pour avoir un enfant. En France, les voies utilisées par les homosexuels voulant accéder à la paternité sont l'adoption, la coparentalité et la gestation pour autrui. Ces modes d'accès à la parentalité ne sont pas ceux autorisés par la loi, mais les modalités envisagées par la plupart des homosexuels français voulant devenir pères. Le recours à une "mère porteuse", par exemple, reste interdit par les lois de bioéthique. En même temps, chaque modèle peut être mis en place au travers de formes variables, notamment la "maternité de substitution", selon qu'on fait appel ou pas à une donneuse d'ovocytes en plus de la mère gestationnelle, et la coparentalité, qui peut varier selon l'orientation sexuelle de la mère et l'implication des différents partenaires, les parents légaux et leurs conjoints respectifs. Mais ces alternatives n'épuisent pas l'éventail des possibilités qui s'offrent à des homosexuels voulant devenir pères. La pratique du transfert d'enfants, par exemple, plus répandue dans des contextes traditionnels, n'est pas répertoriée par les statistiques ou les études concernant la France. ${ }^{12}$

Certains des pères rencontrés en France n'ont eu conscience de l'étendue du choix de modèles qu'en arrivant à l'APGL. Jean, par exemple, ne connaissait pas les coparentalités entre gays et lesbiennes avant d'adhérer à l'APGL. Rencontrer une femme qui accepterait de faire un enfant avec lui, en dehors d'une relation de conjugalité, l'idée lui paraissait “irréaliste", "impossible”. Les premiers contacts avec des mères potentielles, à l'intérieur de l'association, sont restés sans suite. Incrédule sur les possibilités de trouver la bonne personne avec qui construire un projet de coparentalité, Jean s'est tourné vers l'adoption. Néanmoins, il a arrêté la procédure juste avant qu'une décision ne soit rendue. Le psychologue qui l'accompagnait lui aurait fait comprendre que l'adoption n'était pas la solution la mieux adaptée. Jean s'est rendu compte qu'il n'était pas prêt pour renoncer à ses projets de développement professionnel et qu'une adoption était finalement incompatible avec son rythme de vie: "J'avais tout pour le recevoir, les étapes psychologiques, la situation matérielle, ma situation affective, etc., enfin, je pense que j'aurais fait un bon papa adoptif, mais ça m'aurait freiné dans la vie, ça m'aurait causé beaucoup de contraintes". Peu de temps avant d'abandonner la procédure, Jean avait rencontré la femme qui

l 1 Pour une problématisation de la notion de “projet parental”, voir Boltanski (2004) et Delaisi de Parseval (2008).

12 Au Brésil, par exemple, des homosexuels peuvent devenir pères en adoptant des enfants trouvés par des réseaux d'interconnaissance, une pratique liée à la circulation d'enfants dans des milieux populaires (Tarnovski 2002). Bien qu'il s'agisse d'une forme d'adoption, les enfants sont reconnus naturels, ce qui caractérise une pratique illégale dans le contexte brésilien (voir Fonseca 2000). 
allait être la mère de son enfant, ce qui lui a donné une raison de plus pour cesser ses efforts en vue d'une adoption. Finalement, la coparentalité a été pour lui un choix motivé, et pas seulement un choix "par défaut". Tout comme l'adoption, la GPA ne lui convenait pas. Outre le fait qu'il aurait, dans un cas comme dans l'autre, à s'occuper tout seul et à plein temps d'un enfant, la GPA lui donnait l'impression de "priver" un enfant de sa mère. Dans l'adoption, la "mère biologique" de l'enfant n'est pas non plus présente, mais l'adoptant ne se sent pas "responsable” de la séparation. C'est, par exemple, ce que m’a confié un futur père adoptif, justifiant son choix pour l'adoption en même temps que son refus de la GPA. Tant l'adoption que la GPA permettent la création d'une famille centrée sur le couple, mais dans le premier cas les futurs parents n'ont pas l'impression d'être à l'origine d'une rupture des liens ou d'un abandon. Dans le cas de Jean, la coparentalité a permis à la fois de "préserver" le lien mère/enfant et d'avoir suffisamment de temps pour pouvoir se dédier à sa vie professionnelle, dans la mesure où la résidence principale de l'enfant est sans contestation de sa part attribuée à la mère. Cette importance accordée à la figure maternelle fait écho à des représentations plus générales, selon lesquelles le lien de l'enfant à sa mère serait préexistant ou concomitant à la naissance. Ainsi, si d'un côté, la coparentalité peut être considérée comme la voie la plus accessible aux gays voulant être pères, de l'autre ce choix familial peut répondre à une volonté de donner une double référence, paternelle et maternelle, à l'enfant.

En ce qui concerne l'idée que, dans la GPA, les "parents intentionnels"13 induiraient un abandon de l'enfant par sa mère, exprimée entre autres par des pères et des futurs pères adoptifs, les récits de pères ayant effectivement fait appel à la GPA permettent de nuancer cette question. Sans vouloir entrer dans le détail de leur situation familiale et des choix qu'ils ont faits, j'aimerais juste citer quelques éléments pour montrer combien les apparences peuvent être trompeuses. Delaisi de Parseval et Collard (2007) avaient déjà montré que des couples hétérosexuels ayant fait appel à des "mères porteuses" pouvaient, dans certaines circonstances, garder un lien avec la gestatrice de leurs enfants. D'une façon similaire, Sébastien et son compagnon, ainsi que Thierry et Thomas, sont restés en contact avec les gestatrices de leurs enfants, sans complètement écarter l'éventualité d'une rencontre future. ${ }^{14}$ Dans le cas de l'adoption plénière, au contraire, cette possibilité de garder un lien avec les parents d'origine de l'enfant est beaucoup plus rare, malgré les initiatives d'associations en faveur de l' "adoption ouverte" (Fine et Neirinck 2000). En tout cas, aucun des pères adoptifs interrogés, à l'image de ce qui se passe dans la

13 C'est-à-dire, les parents à l'origine du projet d'enfant (voir Delaisi de Parseval et Collard 2007).

14 Indépendamment du fait que, dans ces situations, la gestatrice doit être reconnue comme mère à l'état civil français. 
plupart des adoptions, n'a gardé de contacts avec les parents d'origine de leurs enfants. J'ai même rencontré un futur père adoptif qui, dans l'attente d'aller chercher l'enfant qui lui avait été attribué par l'orphelinat, craignait que sa mère veuille le reprendre avant que la procédure d'adoption ne soit finalisée... En contraste avec ce qui peut se passer dans une situation d'abandon s'achevant par une procédure d'adoption, les surrogate mothers font souvent un travail réflexif leur permettant de ne pas considérer l'enfant qu'elles portent pour un autre couple comme étant le leur (Ragone 1996; Delaisi de Parseval et Collard 2007). A ce sujet, Thierry et Thomas pensent que l'histoire d'un enfant né au moyen d'une GPA est "plus claire" et facile à lui expliquer. Avant de s'engager dans une GPA, le couple avait l'intention d'adopter un enfant, mais ce projet a été abandonné en raison des nombreuses restrictions imposées aux hommes célibataires voulant adopter. ${ }^{15}$ En même temps, ils croyaient qu'en adoptant, ils apporteraient des difficultés additionnelles à un enfant qui, en plus de ne pas connaître ses origines, aurait des parents homosexuels. Ils admettent, néanmoins, que ce raisonnement a été construit rétrospectivement, en contraste avec la vision "idéaliste" du début.

Ces différents arguments pour ou contre l'adoption ou la GPA, exprimés par certains pères, ont en commun le fait de présupposer comme potentiellement problématique l'absence de lien maternel. Dans un cas comme dans l'autre, la mère ou la gestatrice n'est pas présente dans le quotidien des enfants, qui n'ont que des parents masculins, situation que la plupart des homosexuels voulant devenir pères en France ne sont pas prêts à assumer (Cadoret 2001). En ce sens, Martine Gross (2006: 158) remarque que le choix de la coparentalité "[...] apaise, chez les hommes comme chez les femmes, la culpabilité à mettre un enfant au monde dans des conditions inhabituelles". Cela s'exprimerait par l'idée qu'un enfant devrait avoir un père et une mère. ${ }^{16}$

Par ailleurs, la dynamique d'alternance des foyers propre à la coparentalité peut également être perçue comme un avantage. Parallèlement à l'argument

15 Dans la loi française, il n'est pas fait mention de l'orientation sexuelle comme critère pouvant faire obstacle à l'adoption par des célibataires. Les couples homosexuels, en revanche, ne peuvent pas adopter conjointement. Comme le souligne Bruno Perreau (2006), même si certains départements ne s'opposent pas à la délivrance de l'agrément à des candidats se déclarant homosexuels, dans la plupart des cas, ces derniers se voient obligés de cacher leur homosexualité lors des enquêtes sociales. La Cour Européenne des Droits de l'Homme a déjà été saisie à cause de pratiques discriminatoires au motif de l'orientation homosexuelle des candidats à l'adoption. En 2009, le tribunal administratif de Besançon a ordonné au Conseil Général du Jura de délivrer l'agrément à une enseignante qui ne faisait pas secret de son homosexualité.

16 Bien que la conviction exprimée par certains gays et lesbiennes selon laquelle un enfant "doit" avoir un père et une mère soit sans doute marquée par le discours social français sur la "différence des sexes" (Fassin 2008), une idée similaire se retrouve chez certaines femmes ayant fait une IVG (interruption volontaire de grossesse), lorsque le recours à l'avortement est associé à l'idée qu'un enfant ne devrait pas naître "sans père". A ce propos, voir Boltanski (2004: 144-155). 
selon lequel la coparentalité répondrait à une préoccupation de donner un père et une mère à l'enfant, que ce soit pour reproduire un certain modèle de normalité familiale ou en réaction au débat public sur les familles homoparentales, la garde partagée de l'enfant est considérée par certains pères comme un aspect positif des arrangements coparentaux. Tous ne désirent pas un partage égalitaire en ce qui concerne le temps que les enfants passent avec chaque parent. D’une manière générale, les conflits qui peuvent apparaître entre coparents, notamment ceux en rapport avec les modalités d'alternance entre les foyers, concernent plutôt les aspects organisationnels de la coparentalité que son principe, à savoir que la résidence principale de l'enfant soit chez sa mère, ce qui n'est pas systématiquement remis en question. De fait, je n'ai connu aucune coparentalité où la résidence principale ait été fixée chez le père (Tarnovski 2011).

Lanalyse des motivations permet de mieux comprendre comment les acteurs orientent leur choix. Jusqu'ici, ce dernier a été analysé comme étant exclusif, c'est-à-dire qu'en choisissant, on ne retient qu'une possibilité. Mais rien n'empêche que ce choix puisse porter sur plus d'une option. Cela a été le cas de Vincent et Victor, le premier ayant à la fois adopté et fait une coparentalité, et les deux enfants étant arrivés presque en même temps. Mais l'idée que l'on puisse faire un choix multiple n'a pas laissé indifférent leur entourage d'amis homosexuels: "Cela paraissait farfelu aux yeux de certaines personnes, de mélanger coparentalité et adoption. [...] Pour certains on ne mélange pas tout. Pour certains. Moi, ça ne me paraissait pas si incompatible" [Vincent]. La réaction d'autres parents homosexuels vis-à-vis de cette situation semble indiquer l'existence d'une division de l'univers des familles homoparentales par des frontières internes et invisibles séparant chaque catégorie de famille comme autant d'alternatives qui ne devraient pas se "mélanger". Le récit d'un autre père donne une indication des enjeux éventuels d'un tel "mélange". Bruno, en même temps qu'il se lançait dans une coparentalité avec son compagnon Bernard, a fait une demande d'agrément d'adoption. Bien que la décision lui ait été favorable, il n'était plus sûr de vouloir continuer ces démarches, car le projet d'adoption "ne faisait pas écho" chez Bernard. Lidée de créer une asymétrie à l'intérieur d'une éventuelle fratrie instaurée par les différents modes d'arrivée des enfants le gênait. Bernard s'est dit “ [...] perplexe face à la perspective d'avoir à la fois, à la maison, un enfant adopté mais qui sera là tout le temps et un enfant, ou des enfants, qui sont en alternance et qui ne viennent que de temps en temps". Ce malaise face au "mélange" des modalités d'arrivée des enfants tient aussi aux spécificités concernant l'établissement de la filiation. L'adoption s'appuie sur une valorisation de la volonté et de la reconnaissance sociale comme fondements de la filiation et de l'amour parental, tandis que la coparentalité peut réactiver des représentations biogénétiques de la parenté. Chaque modalité aurait ainsi des spécificités concernant le 
fondement de la filiation qui, du point de vue de certains parents, ne seraient pas conciliables dans une même configuration familiale.

Outre les raisons multiples pouvant orienter le sujet, le choix du mode d'accès à la paternité et de la configuration familiale résulte également d'un aménagement des attentes initiales et des ressources financières, de temps ou relationnelles que l'on est capable de réunir. En ce sens, le choix en question peut être influencé par la situation socioprofessionnelle ou le lieu de résidence, par exemple. Ceux qui habitent à Paris ont à leur disposition un réseau d'entraide, grâce à l'APGL, que n'ont pas ceux habitant en province. Cette association, par l'action de ses membres, offre des espaces de réflexion permettant au nouvel arrivant d'élaborer avec plus de clarté le projet parental qui lui convient le mieux. Ceux qui choisissent l'adoption ou la GPA peuvent participer à des forums de discussion favorisant l'échange d'informations, parfois précieuses, pour mener à bien leurs projets.

Certaines activités de l'APGL peuvent être destinées à faciliter la réalisation du projet, comme c'est le cas des annonces publiées dans le journal interne ou des réunions conviviales permettant de faire se rencontrer futurs pères et futures mères en quête de partenaires pour une coparentalité. Dans les situations où la réalisation d'une coparentalité, par exemple, s'est faite par des annonces publiées sur des sites Internet sans lien avec l'APGL ou à travers une rencontre non "provoquée", l'association peut apporter de l'aide concernant les points sur lesquels réfléchir avant de "partir à l'action" ou même après (Gross et Peyceré 2005). Parallèlement à l'APGL, d'autres réseaux se sont constitués pour aider des homosexuels voulant être parents, comme par exemple le groupe de pères adoptifs dont les membres se retrouvent au moins une fois par an et auquel j'ai été présenté par l'un des mes interlocuteurs. Sans aucun lien direct avec l'APGL, ce groupe s'est constitué sur la base d'une expérience partagée, celle d'hommes officiellement célibataires ayant adopté leurs enfants dans un même pays. Si, d'une part, ce groupe permet aux enfants de se forger des références communes, en tant que fils et filles adoptives de parents homosexuels et par le partage d'une même origine culturelle, ${ }^{17}$ de l'autre il est devenu une référence pour d'autres homosexuels, en couple ou célibataires, voulant adopter des enfants et en manque de soutien pour les aider dans leurs démarches ou tout simplement cherchant un espace de convivialité et de dialogue. Certes, des associations ayant pour finalité d'aider des couples hétérosexuels voulant adopter ou réaliser une GPA, avant que celle-ci ne soit interdite en France, existent ou ont existé dans le contexte français. En contraste avec ces initiatives, l'APGL ou les groupes informels de parents homosexuels ne limitent pas leur champ d'action aux aspects pratiques liés à la réalisation d'un

17 Plus encore qu'une même origine culturelle, certains enfants sont passés par le même orphelinat, de sorte qu'ils se connaissaient déjà avant d'être adoptés par des pères "célibataires" français. 
projet parental. Ce qui les distingue en premier lieu, mais non exclusivement, c'est de rendre possibles ces projets parentaux, en conjuguant positivement homosexualité et parentalité.

\section{CONSIDÉRATIONS FINALES}

Dans cet article, j'ai analysé comment le désir d'enfant prend forme et s'exprime dans certains contextes sociaux actuels, en prenant l'exemple de pères homosexuels français. La construction de ce désir, phénomène très contemporain selon certains auteurs (Godelier 2004; Delaisi de Parseval 2008), pourrait être analysée dans n'importe quel groupe ou catégorie sociale. Néanmoins, le discours militant sur le sujet, tout comme certaines analyses, semblent doter ce désir d'une réalité universelle, voire anhistorique. A l'intérieur de ce discours, ce n'est pas l'existence du désir d'enfant qui demanderait une explication mais son absence. Dans ce raisonnement, si les familles homoparentales ne sont apparues que très récemment dans la plupart des sociétés occidentales, c'est qu'auparavant ces mêmes sociétés s'opposaient et érigeaient des obstacles à la réalisation de ce désir. Ce discours assume l'homosexualité, en tant qu'attribut de l'individu, comme une donnée qui n'est pas discutée dans son historicité. Cette approche problématise le regard social sur l'homosexualité, mais non pas sa construction sociale et historique, de manière à mettre en perspective les particularités des familles homoparentales occidentales contemporaines. L'analyse des récits des pères gays montre, au contraire, que ce désir prend forme collectivement, révélant ainsi que son existence subjective n'est pas indépendante du contexte social dans lequel se trouve l'individu. Mon hypothèse est que les associations et les réseaux de familles homosexuelles aident les homosexuels voulant devenir parents à réaliser leurs projets parentaux, mais encore que la visibilité de l'homoparentalité qu'ils ont contribué à produire participe à la création du désir d'enfant chez les homosexuels, dans la mesure où l'homoparentalité devient une réalité possible.

La possibilité contemporaine d'un désir d'enfant exprimé par des gays et des lesbiennes, en tant que tels, est révélatrice non seulement des changements dans le domaine de la famille et de la parenté, mais aussi des processus socioculturels, historiques et politiques de construction de la sexualité, qui l'ont cristallisé comme une dimension fondamentale dans la définition de l'individu occidental moderne (Foucault 1994). Mais le fait de montrer leur historicité n'affaiblit pas la force sociale et politique de ces constructions de la sexualité et du désir d'enfant et moins encore l'importance de ces réalités pour le sujet.

Certes, tous les homosexuels ne veulent pas devenir parents dans ce contexte de visibilité des familles homoparentales. C'est alors que les aspirations individuelles, l'histoire personnelle et la subjectivité trouvent toute leur place pour expliquer pourquoi le désir d'enfant est plus fort chez certains, et 
inexistant chez d'autres. Néanmoins, ce que j'ai essayé de montrer est que, dans le contexte français, et plus largement européen, les homosexuels sont de plus en plus amenés à faire des choix à propos de cette question. Progressivement, le fait de ne pas vouloir d'enfants cesse d'être une évidence, pour devenir un choix. L'homoparentalité est devenue une réalité socialement visible, permettant aux jeunes générations, mais parfois aussi aux anciennes, d'exprimer un désir d'enfant. Cette visibilité des familles homoparentales n'est pas sans déranger certains homosexuels un peu plus âgés, qui se voient confrontés aux "choix" qu'ils ont faits ou non, justement, dans leurs vies. Certes, tous n'ont pas à leur disposition les mêmes moyens pour pouvoir réaliser un projet parental et accueillir un enfant. Pour une partie de la population homosexuelle, ne pas avoir d'enfant n'est pas simplement le résultat d'un choix conscient, mais une réalité à laquelle ils ont dû se résigner. De même, il faut évidemment considérer le rôle pionnier d'une partie de la population homosexuelle qui, contre toute attente, a poursuivi sa volonté de fonder une famille en ayant ou en adoptant des enfants, ouvrant ainsi la voie pour que d'autres puissent envisager des projets similaires et avoir à leur disposition des soutiens pour les réaliser. La revendication et la mise en pratique d'une conjugaison positive entre paternité et homosexualité, ainsi que sa traduction en un discours social de plus en plus visible, ont participé à une reformulation de ce que "être" homosexuel veut dire.

\section{BIBLIOGRAPHIE}

ALMEIDA, Miguel Vale de, 2010, A Chave do Armário: Homossexualidade, Casamento, Família. Florianópolis, Ed. da UFSC.

BOLTANSKI, Luc, 2004, La condition fectale. Paris, Gallimard. BOZON, Michel, 2002, Sociologie de la sexualité. Paris, Nathan.

CADORET, Anne, 2001, "Etre père sans femme: la paternité gay", TSANTSA - Revue de la

Société Suisse d'Ethnologie, 6: 83-92.

— 2002, Des parents comme les autres: Homosexualité et parenté. Paris, Éditions Odile Jacob. CADOrET, Anne, Martine Gross, Caroline MÉCARY, et Bruno PERREAU (eds.), 2006,

Homoparentalités: Approches scientifiques et politiques. Paris, PUF.

DELAISI DE PARSEVAL, Geneviève, 2008, Famille à tout prix. Paris, Seuil.

DELAISI DE PARSEVAL, Geneviève, et Chantal COLLARD, 2007, "La gestation pour autrui:

un bricolage des représentations de la paternité et de la maternité euro-américaines”, L'Homme, 183: 29-54. 
DESCOUTURES, Virginie, 2008, Les mères lesbiennes: Contribution à une sociologie de la parentalité. Paris, Université René Descartes (Paris V), thèse de doctorat en sociologie.

FASSIN, Éric, 2008, L'inversion de la question homosexuelle. Paris, Éditions Amsterdam.

FAVRET-SAADA, Jeanne, 1999, “Enfin au-delà du PaCS”, ProChoix, 12: 15-17.

FINE, Agnès, et Claire NEIRINCK (eds.), 2000, Parents de sang, parents adoptifs: Approches juridiques et anthropologiques de l'adoption. France, USA, Canada, Europe. Paris, LGDJ.

FONSECA, Claudia, 2000, "La circulation des enfants pauvres au Brésil : une pratique locale dans un monde globalisé”, Anthropologie et Sociétés, 24 (3): 53-73.

FOUCAULT, Michel, 1994, Histoire de la sexualité: La volonté de savoir. Paris, Gallimard.

FOURNIER, Sandrine, 2003, Enquête sur l'homoparentalité dans la Baie de San Francisco.

Toulouse, EHESS, mémoire de DEA en anthropologie sociale et historique.

GODELIER, Maurice, 2004, Métamorphoses de la parenté. Paris, Fayard.

GRATTON, Emmanuel, 2006, L'homoparentalité, côté pères. Paris, Université Denis Diderot (Paris VII), thèse de doctorat en sociologie.

GROSS, Martine (ed.), 2005, Homoparentalités: État des lieux. Paris, Érès.

— 2006, "Désir d'enfant chez les gays et les lesbiennes”, Terrain, 46: 151-164.

GROSS, Martine, et Mathieu PEYCERÉ, 2005, Fonder une famille homoparentale. Paris, Ramsay.

GROSS, Martine, et Dominique Mehl, 2011 , “Homopaternités et gestation pour autrui", Enfances, Familles, Générations, 14: 95-112.

Grossi, Miriam, Anna Paula UZIEL, et Luiz MELlO (eds.), 2007, Conjugalidades, Parentalidades e Identidades Lésbicas, Gays e Travestis. Rio de Janeiro, Garamond.

HERBRAND, Cathy, 2009, "Déclinaisons du désir d'enfant dans les coparentalités homosexuelles", Revue des Sciences Sociales, 41: 42-51.

LEWIN, Ellen, 1993, Lesbian Mothers: Accounts of Gender in American Culture. Ithaca/Londres, Cornell University Press.

—, 2009, Gay Fatherhood: Narratives of Family and Citizenship in America. Chicago, University of Chicago Press.

PERREAU, Bruno, 2006, “Les organismes autorisés pour l'adoption:analyse d'une délégation de service public", en Anne Cadoret, Martine Gross, Caroline Mecary et Bruno Perreau (eds.), Homoparentalités: Approches scientifiques et politiques. Paris, PUF, 163-173.

RAGONE, Helena, 1996, "Chasing the blood tie: surrogate mothers, adoptive mothers and fathers", American Ethnologist, 23 (2): 352-365.

SCHNEIDER, David M., 1997, "The power of culture: notes on some aspects of gay and lesbian kinship in America today”, Cultural Anthropology, 12 (2): 270-274.

TAIN, Laurence, 2005, "Um filho quando eu quiser? O caso da França contemporânea", Revista Estudos Feministas, 13 (1): 53-67.

TARNOVSKI, Flávio L., 2002, "Pais Assumidos": Adoção e Paternidade Homossexual no Brasil Contemporâneo. Florianópolis, UFSC, mémoire de "mestrado" en anthropologie sociale.

—_, 2010, Etre père et homosexuel dans la France contemporaine. Toulouse, EHESS, thèse de doctorat en anthropologie sociale.

— 2011 , "Les coparentalités entre gays et lesbiennes en France: le point de vue des pères", Vibrant, 8 (2): 140-163.

—, 2012, "Paternité et sexualité dans la construction de soi", Ethnologie Française, 42 (1): 145-153.

THÉRY, Irène, 1998, Couple, filiation et parenté aujourd'hui. Paris, Odile Jacob. 